\title{
Immediate Effect of Bilateral Talocrural Joint Manipulation on Postural Balance in Healthy Subjects
}

Paes FJV', da Silva RA ${ }^{2,3}$, Freira ALG ${ }^{1}$, Politti $F^{4}$, Chiavegato $L^{1}$, Geraldes $A R^{5}$ and Amorim CF $^{1 *}$

${ }^{1}$ Physical Therapy Program, Universidade Cidade de São Paulo (UNICID), São Paulo- SP, Brazil

${ }^{2}$ Center for Health Science Research, Laboratory of Functional Evaluation and Human Motor Performance, Universidade Norte do Paraná (UNOPAR), Londrina-PR, Brazil

${ }^{3}$ Masters and Doctoral Program in Rehabilitation Sciences UEL/UNOPAR, Londrina-PR, Brazil

${ }^{4}$ Rehabilitation Sciences Department, Universidade Nove de Julho (UNINOVE), São-Paulo, SP, Brazil

${ }^{5}$ Physical Education Department, Universidade Federal de Alagoas (UFAL), Maceio, AL, Brazil

\begin{abstract}
The aim of this study was to assess the immediate effect of bilateral talocrural joint manipulation on the postural balance of healthy subjects. Ninety healthy subjects ( 21 males and 69 females) were included in this study. The mean age, height and body weight of the subjects were 22.21 years, $165.1 \mathrm{~cm}$ and $62.58 \mathrm{Kg}$, respectively. Following collection of anthropometric data, the subjects were allocated randomly into an experimental group (receiving real talocrural joint manipulation), a placebo group and a control group. Before and after manipulation, the subjects performed two postural balance tasks, inquiet standing position (eyes open and eyes closed) on a force platform. The stabilometric data were recorded for 60 seconds in each condition. Sway area, medio-lateral (ML) and anteroposterior (AP) velocities and median frequency were calculated from the center of pressure (COP) displacements. The results showed that in the experimental group the COP sway velocity decreased in both directions of movement (AP and $\mathrm{ML}$ ) and in both visual tasks (eyes open and eyes closed). In general, the placebo and control groups had similar postural balance across balance parameterss. Significant effect of the bilateral talocrural joint manipulation was found on main balance parameter like COP velocity in the anteroposterior and mediolateral directions. These results have some much implications for manual therapy rehabilitation of individuals with ankle instability.
\end{abstract}

Keywords: Posture; Manual therapy; Physical Therapy; Rehabilitation

\section{Introduction}

Balance is the ability to maintain equilibrium by positioning the centre of gravity over the base of support of body. It is carried out by a complex process involving afferents from the sensory system, integration of efferents by central nervous system and afferent being sent to an intact musculoskeletal system [1].

Static position sense, or the perception of a stationary joint angle, depends on afferent information from joint receptors along with signals from muscle spindles and Golgi tendon organs [2]. Ruffini receptors and Pacinian corpuscles are both classified as dynamic receptors; however, Ruffini receptors have also been described as static receptors based on their low-threshold, slow-adapting characteristics. It has been suggested that these types of receptors are stimulated when a joint is moved near the end range of motion [3]. Therefore, it is suggested that good proprioception is of concern in promoting of dynamic joint and of functional stability in sports, activities of daily living and occupational tasks.

The ankle joints also play an important role in maintaining postural stability [4]. Loss of postural control has been consistently observed in patients with chronic ankle instability [5] and after an acute ankle sprain [6], which can present poor proprioception and/or poor muscular capacity to protect the joint and physical performance. Ankle manipulation has excellent potential to assist in the treatment of a variety of foot disorders related to postural instability. The understanding of the techniques of ankle manipulation as well as of the recommendations for its use can be a clinical valuable tool for the rehabilitation programs. Some scientific evidences has showed positive effects of joint mobilization on functional balance performance related to changes in sensorimotor system function $[7,8]$. However, functional tests can measure balance deficit indirectly through the recorded timelimit of physical performance (i.e., one aspect of physical condition or muscular capacity of individual), while centre of pressure (COP) parameters from a force platform can directly analyze balance deficits related to proprioception and postural adjustments (feedback and feedfoward) of the neuromuscular system [9]. This is further supported by a recent study [10] suggesting that clinical functional tests such as time-limit to stand one leg and laboratory-based measures from COP parameters may capture different aspects of balance.

Another aspect of these two studies [7,8] was reporting the results with only massage and simple joint mobilization on intervention processus. Few studies has investigated the caudal talocrural joint manipulation from high-velocity and low-amplitude caudal thrust. Comparing with placebo, López-Rodríguez et al. [11] that investigated the caudal talocrural joint manipulation in athletic individuals with grade II ankle sprain showed better balance related to load support at foot level. Altough these effects support, at least, positive results on postural system regulation, it is unclear still whether there is an immediate (acute session) effect on postural stability after therapeutic ankle manipulation during a standing position with two different conditions (eyes open and eyes closed) on a force platform measurement.

Postural stability has traditionally been examined by spatial

*Corresponding author: Amorim CF, Physical Therapy Program, Universidade Cidade de São Paulo - UNICID, Rua Cesário Galeno 448/475, 03071-000 Tatuapé, Brazil, E-mail: cesar@emgsystem.com.br

Received June 25, 2013; Accepted October 24, 2013; Published October 31, 2013

Citation: Paes FJV, da Silva RA, Freira ALG, Politti F, Chiavegato L, et al. (2013) Immediate Effect of Bilateral Talocrural Joint Manipulation on Postural Balance in Healthy Subjects. J Ergonomics 3: 122. doi:10.4172/2165-7556.1000122

Copyright: $\odot 2013$ Paes FJV, et al. This is an open-access article distributed unde the terms of the Creative Commons Attribution License, which permits unrestricted use, distribution, and reproduction in any medium, provided the original author and source are credited. 
measures of the center of pressure (COP), where greater COP movements are considered signs of postural instability. COP parameters are defined as the coordinates of the resultant force applied through the feet on a force plate along anteroposterior (AP) and mediolateral (ML) orthogonal axes [12]. The stabilometric parameters that are most commonly reported in the literature describe the statistical properties of the COP trajectory, which is considered a stationary signal, in the time and frequency domains $[13,14]$. The most commonly used COP parameters are mean velocity and sway area, with the mean velocity considered the most reliable $[15,16]$.

Although one study found that talocrural joint manipulation immediately affects on postural stability [7], a similar effect was not observed in another study [12]. These discrenpacies in the results may be related to methodological differences regarding protocol or equipment (functional performance, balance scale, force platform baropodometer, functiona), patient age (adults versus elderly) and patient activity level (athletes and non-athletes). The aim of this study was thus to assess immediate effect of bilateral talocrural joint manipulation on the postural balance of healthy subjects during a standing position in two tasks (eyes open and eyes closed) on a force platform, which is of concern to determine the effects of intervention on biomechanical and neuromuscular strategies of postural control.

\section{Materials and Methods}

\section{Subjects}

Ninety healthy subjects ( 21 males and 69 females) were recruited voluntary and by convenience to participate of this study. The subjects characteristics were in mean age 22.21 years (SD 2.42), height 165.1 $\mathrm{cm}$ (SD 0.7) and body weight $62.58 \mathrm{Kg}$ (SD 12.70). An orthopedic physician examined all subjects before each trial to ensure that they had no musculoskeletal injuries, neuromuscular disorders, lower extremity injuries, spine pathology, balance problems or pain. Subjects reporting an acute ankle sprain within the past 6 months, a previous history of lower extremity surgeries or a fracture within the past 6 months were also excluded from the study.

Following the collection of anthropometric data, the subjects were allocated randomly by using a computer software into an experimental group, a placebo group and a control group (Table 1). All subjects were informed about the experimental protocol and the potential risks of the study and gave written consent before their participation. The protocol and the consent form had been previously approved by the local Ethics committee (UNICID, SP).

\section{Procedure and data collection}

The bilateral talocrural joint manipulation procedure in the experimental group was similar to that described by López-Rodríguez et al. [11]. A trained therapist applied a high-velocity, low-amplitude caudal thrust directed at the talocrural joint in the following manner: with the patient in the supine position, the therapist wrapped his or her hands around the leg with the fingers at the level of the neck of the talus and then applied caudal traction, increasing the dorsiflexion at the talocrural joint. In the placebo group, the positioning of the physical therapist and the subjects remained the same, but the handgrip was such that neither therapeutic traction nor joint manipulation occurred: the volunteers feet were simply held bilaterally for 20 seconds and then repositioned on the table. For both intervention and placebo groups the same therapist applied the technique to avoid possible bias. In the control group, after the first data recording, the volunteers remained lying on the table for one minute and stabilometric data recording recommenced.

\section{Stabilometric data collection}

Before testing, all subjects were familiarized with the balance protocol. Two conditions were tested during quiet standing: (i) eyes open and eyes closed in pre-manipulation of talocrural joint; (ii) eyes open and eyes closed after manipulation of talocrural joint occurred. These two conditions were performed before the (pre) and immediately after (post) the manipulation without any resting time. The order of the trials was randomized for each subject. Another investigator trained conducted all balance evaluation on the force platform (e.g. evaluator blind to intervention). The participant remained with arms alongside the body and gaze fixed on a circular target $(5 \mathrm{~cm}$ in diameter) at the height of the glabellum attached to a pedestal at a distance of 1.5 meters from the volunteer. The stabilometric data were recorded for 60 seconds at a sampling frequency of $100 \mathrm{~Hz}$ in each trial, with the feet close together on a BIOMEC 400 16-bit force plate (EMG System do Brasil'). This platform features load cells with an internal circuit that changes electrical resistance upon the application of a force; the device's accuracy is $0.5 \%$ and its dimensions are $500 \times 500 \mathrm{~mm}$.

The data analyses were performed using Matlab 7.1 software (The Math Works, Inc. Natick, MA, USA). The COP data were low-pass filtered at $10 \mathrm{~Hz}$ with a Butterworth filter. After filtering, the COP sway area was calculated, as well as the mean velocity and frequency of the COP data in the anteroposterior and mediolateral directions. The COP excursion was defined as the maximum displacement of the COP in each direction (i.e., the distance between the farthest points in the COPap and COPml directions). The CoP area was estimated by fitting an ellipse to the CoP data (AP versus ML) that encompasses $95 \%$ of the data [17]. The COP velocity was calculated as the total COP displacement divided by the measurement time. The frequency of the COP displacement is the frequency below which $80 \%$ of the COP spectral power occurs $[18,19]$. The power spectral density of the COP data was estimated by the Welch periodogram method with a resolution of $0.039 \mathrm{~Hz}$. The validity and reliability of all these parameters computed with this BIOMEC 400 force platform have been accepted for both young adults and elderly subjects as reported by a recent study [17].

\section{Statistical analyses}

The statistical data was analyzed in SPSS 16.0 for Windows (SPSS Inc., Chicago, IL, USA); the Shapiro-Wilk test was used to check for normal data distribution. Since the data were not normally distributed, the Kruskal-Wallis test was used to test the homogeneity of stabilometric parameters (sway area, velocity and median frequency) between the three groups (experimental, placebo and control) before manipulation of the talocrural joint. The manipulation effect of the talocrural joint in the two conditions visual (eyes open and eyes closed)

\begin{tabular}{|c|c|c|c|}
\hline & $\begin{array}{c}\text { Experimental Group } \\
(\mathbf{n = 3 0 )}\end{array}$ & $\begin{array}{c}\text { Placebo Group } \\
\mathbf{( n = 3 0 )}\end{array}$ & $\begin{array}{c}\text { Control Group } \\
(\mathbf{n}=\mathbf{2 9})\end{array}$ \\
\hline Gender & $5 \mathrm{M} / 25 \mathrm{~F}$ & $7 \mathrm{M} / 23 \mathrm{~F}$ & $8 \mathrm{M} / 21 \mathrm{~F}$ \\
\hline Age (yrs) & $22.20 \pm 2.64$ & $22.07 \pm 2.69$ & $22.45 \pm 1.93$ \\
\hline Body weight (Kg) & $61.42 \pm 8.91$ & $61.06 \pm 11.36$ & $65.12 \pm 16.89$ \\
\hline Height (m) & $1.64 \pm 0.07$ & $1.65 \pm 0.08$ & $1.67 \pm 0.08$ \\
\hline
\end{tabular}

The data are presented in mean values with Standard deviation ( \pm ). M: male; F: female 
Citation: Paes FJV, da Silva RA, Freira ALG, Politti F, Chiavegato L, et al. (2013) Immediate Effect of Bilateral Talocrural Joint Manipulation on Postural Balance in Healthy Subjects. J Ergonomics 3: 122. doi:10.4172/2165-7556.1000122

Page 3 of 5

conditions was determined by the Wilcoxon test for each group of subjects (experimental, placebo and control) with an alpha level of 0.05 .

\section{Results and Discussion}

To analyse the effect of talocrural joint manipulation, it was necessary to verify whether the stabilometric data presented significant differences between groups (experimental, placebo, control) before the intervention. There were no significant differences in the values for sway area, velocity and median frequency in the COPap and COPml displacement between the groups in both balance experimental condition (eyes open and eyes closed) (Table 2).

In relation to interventions effects on balance after the manipulation, experimental group reduced in mean $0.4 \mathrm{~cm} / \mathrm{s}$ the sway COP velocity values in the two directions (AP and ML) in both tested conditions (Table 3). The median frequency was significantly lower in the experimental group after manipulation at the eyes open condition only. These results support the improve of postural stability on experimental manipulation group. In general, similar postural balance results were found between placebo and control groups across balance parameters. Interestingly, the control group poored the balance of $0.34 \mathrm{~cm}^{2}$ from sway COP area results for eyes open condition (Table 3).

The results of the present study are in agreement with previous studies, despite the protocols and sample differences. Similar effects have been described after caudal talocrural joint manipulation [11] and an association of massage and mobilization of the feet and ankles $[7,8]$ Apparently, high-velocity and low-amplitude caudal thrust directed at the talocrural joint helps to improve the balance by stimulating the afferents inputs arising of mechanoreceptors in the ankle ligaments and capsule. These changes were only observed in the experimental group and demonstrate that change occurs in sensorimotor system function following joint mobilization. Muscle spindles play a large role in kinesthesia and provide information about muscle length changes, while joint receptors respond primarily at the end ranges of movement [20]. Thus, stimulating the mechanoreceptors of the ankle joint by this type of manipulation may lead to an increase in neuromuscular activity

\begin{tabular}{|c|c|c|c|c|}
\hline & Experimental Group & Placebo Group & Control Group & P-Value \\
\hline \multicolumn{5}{|l|}{ Eyes Open } \\
\hline Area $\left(\mathrm{cm}^{2}\right)$ & $1.55 \pm 0.80$ & $1.37 \pm 0.90$ & $1.47 \pm 0.64$ & 0.27 \\
\hline Velocity COPap(cm/s) & $0.66 \pm 0.11$ & $0.64 \pm 0.08$ & $0.69 \pm 0.12$ & 0.12 \\
\hline Velocity COP ml (cm/s) & $0.58 \pm 0.09$ & $0.54 \pm 0.08$ & $0.56 \pm 0.09$ & 0.21 \\
\hline Median Frequency COPap(Hz) & $0.15 \pm 0.06$ & $0.15 \pm 0.06$ & $0.17 \pm 0.06$ & 0.48 \\
\hline Median Frequency COPml (cm) & $0.22 \pm 0.08$ & $0.26 \pm 0.09$ & $0.23 \pm 0.07$ & 0.31 \\
\hline \multicolumn{5}{|l|}{ Eyes Closed } \\
\hline Area $\left(\mathrm{cm}^{2}\right)$ & $2.61 \pm 2.32$ & $1.66 \pm 0.76$ & $2.19 \pm 1.11$ & 0.38 \\
\hline Velocity COPap(cm/s) & $0.83 \pm 0.17$ & $0.78 \pm 0.16$ & $0.67 \pm 0.17$ & 0.11 \\
\hline Velocity COP ml (cm/s) & $0.65 \pm 0.13$ & $0.60 \pm 0.11$ & $0.64 \pm 0.10$ & 0.13 \\
\hline Median Frequency COPap $(\mathrm{Hz})$ & $0.19 \pm 0.08$ & $0.21 \pm 0.05$ & $0.21 \pm 0.06$ & 0.49 \\
\hline Median Frequency COP $\mathrm{ml}(\mathrm{cm})$ & $0.22 \pm 0.06$ & $0.26 \pm 0.08$ & $0.22 \pm 0.06$ & 0.28 \\
\hline
\end{tabular}

Mean and standard deviation (SD) values of the variables sway area of COP, velocity and frequency in both directions (AP and ML). The Kruskall-Wallis test was performed to compare the estabilometric parameters between the three groups (experimental, placebo and control groups) before talocrural joint manipulation. $P>0.05$ : no significant difference between-group.

Table 2: Comparison between the three groups on postural stability task during the two conditions (eyes open and eyes closed) before manipulation of joint.

\begin{tabular}{|c|c|c|c|c|c|c|}
\hline & \multicolumn{2}{|c|}{ Eyes Open (Mean \pm SD) } & \multirow[b]{2}{*}{$P$-Value } & \multicolumn{2}{|c|}{ Eyes Closed (Mean \pm SD) } & \multirow[b]{2}{*}{$P$-Value } \\
\hline & $\mathrm{T1}$ & T2 & & T1 & T2 & \\
\hline \multicolumn{7}{|l|}{ Experimental group } \\
\hline Area $\left(\mathrm{cm}^{2}\right)$ & $1.55 \pm 0.80$ & $1.72 \pm 1.01$ & 0.19 & $2.61 \pm 2.32$ & $2.09 \pm 1.08$ & 0.22 \\
\hline Velocity COPap (cm/s) & $0.66 \pm 0.11$ & $0.62 \pm 0.09$ & $<0.001^{*}$ & $0.83 \pm 0.17$ & $0.78 \pm 0.17$ & $0.001^{*}$ \\
\hline Velocity COPml (cm/s) & $0.58 \pm 0.09$ & $0.54 \pm 0.09$ & $<0.001^{*}$ & $0.65 \pm 0.13$ & $0.61 \pm 0.13$ & $0.001^{*}$ \\
\hline Median Frequency COPap (Hz) & $0.15 \pm 0.06$ & $0.16 \pm 0.05$ & 0.53 & $0.19 \pm 0.08$ & $0.19 \pm 0.07$ & 0.64 \\
\hline Median Frequency COPml (Hz) & $0.22 \pm 0.08$ & $0.18 \pm 0.06$ & $0.01^{*}$ & $0.22 \pm 0.06$ & $0.21 \pm 0.07$ & 0.31 \\
\hline \multicolumn{7}{|l|}{ Placebo group } \\
\hline Area $\left(\mathrm{cm}^{2}\right)$ & $1.37 \pm 0.90$ & $1.32 \pm 0.73$ & 0.88 & $1.66 \pm 0.76$ & $1.72 \pm 0.82$ & 0.27 \\
\hline Velocity COPap (cm/s) & $0.64 \pm 0.08$ & $0.62 \pm 0.09$ & 0.06 & $0.78 \pm 0.16$ & $0.75 \pm 0.14$ & 0.12 \\
\hline Velocity COPml (cm/s) & $0.54 \pm 0.08$ & $0.52 \pm 0.08$ & 0.06 & $0.60 \pm 0.11$ & $0.59 \pm 0.09$ & 0.11 \\
\hline Median Frequency COPap (Hz) & $0.15 \pm 0.06$ & $0.16 \pm 0.05$ & 0.34 & $0.21 \pm 0.05$ & $0.20 \pm 0.05$ & 0.48 \\
\hline Median Frequency COPml (Hz) & $0.26 \pm 0.09$ & $0.22 \pm 0.07$ & $0.03^{*}$ & $0.26 \pm 0.08$ & $0.24 \pm 0.09$ & 0.06 \\
\hline \multicolumn{7}{|l|}{ Control group } \\
\hline Area $\left(\mathrm{cm}^{2}\right)$ & $1.47 \pm 0.64$ & $1.81 \pm 0.93$ & $0.004^{*}$ & $2.19 \pm 1.11$ & $2.48 \pm 1.76$ & 0.38 \\
\hline Velocity COPap (cm/s) & $0.69 \pm 0.12$ & $0.66 \pm 0.10$ & 0.08 & $0.67 \pm 0.17$ & $0.66 \pm 0.17$ & 0.14 \\
\hline Velocity COPml (cm/s) & $0.56 \pm 0.09$ & $0.55 \pm 0.09$ & 0.19 & $0.64 \pm 0.10$ & $0.64 \pm 0.14$ & 0.13 \\
\hline Median Frequency COPap (Hz) & $0.17 \pm 0.06$ & $0.15 \pm 0.07$ & 0.34 & $0.21 \pm 0.06$ & $0.21 \pm 0.06$ & 0.49 \\
\hline Median Frequency COPml (Hz) & $0.23 \pm 0.07$ & $0.19 \pm 0.06$ & $0.01^{*}$ & $0.22 \pm 0.06$ & $0.23 \pm 0.05$ & 0.88 \\
\hline
\end{tabular}

Means and standard deviation (SD) values are presented.

* Significant differences between times measures from Wilcoxon test in each group from each COP parameter analysis.

Table 3: The influence of manipulation of the talocrural joint, in the time 1 and 2 (T1 and T2) on COP parameters for two conditions (eyes open and eyes closed) 
and enhanced joint stability through co-activation of the musculature surrounding the joint. This hypothesis can be futher supported from a combination of diminished proprioception and increasing muscle weakness when related as possible causes of chronic ankle instability [21]. Moreover, the effects of lateral ankle ligament anesthesia on time-to-boundary measures of single limb postural control in healthy adults are similar to deficits found in individuals with chronic ankle instability [22,23].

Postural balance control depends on the integrity of the visual, vestibular and mechanoreceptor feedback regarding the lower limbs. Any malfunction or improper feedback from these receptors would cause inappropriate motor reaction and poor balance control [19].

Parameters that describe well COP excursions are frequently used to measure stability and discriminate pathological mechanisms. This is possible since the COP signal is proportional to ankle torque, a combination of descending motor commands as well as mechanical properties of the surrounding musculature. Thus, the results obtained by means of stabilometric recording demonstrated that talocrural joint manipulation produces an immediate effect on the velocity of body sway in quiet standing in both conditions (eyes open and eyes closed). According to a recent review of the test-retest reliability of COP measures in bipedal static task conditions, the reliability of the eyes closed condition is greater than that of the eyes open condition, and the mean velocity is one of the most commonly used (and the most reliable) COP parameter [16]. This parameter was also the most sensitive to discriminate the manipulation effects in the two directions (AP and ML) in both conditions. A recent study [17] also pointed out the reliability of this parameter for both young adults and elderly subjects, both healthy, during a challenging balance tasks such as unipodal condition.

The manipulation of the talocrural joint can provide mechanical benefits for postural control. Therefore, manipulation interventions can be combined with lower limb proprioceptive training programs and other rehabilitation strategies such as balance training. Such a combination of therapies may lead to greater improvement in selfreported function and changes in static postural control. In addition, clinical measurements of stabilometric parameters can be used to track progress during the rehabilitation process.

Finally, the overall results of this study cannot necessarily be generalized to all subjects, since they may not represent the full heterogeneity of balance status among the patients with ankle disorders. Another limitation is the use of stading position on a force platform that not reveal all functionality of limb, altougth the use of the two conditions of postural control assessment (eyes open and eyes closed). Furthermore, a complementary analysis of muscular activity using electromyography simultaneous to force platform parameters computation and time reaction would be relevant to help interpret the results.

In conclusion, the significant effect of the bilateral talocrural joint manipulation wasa found on main balance parameter like COP velocity in the anteroposterior and mediolateral directions. These results have some much implications for manual therapy rehabilitation of individuals with ankle instability.

\section{Clinical Messages}

- Immediate bilateral talocrural joint manipulation is efficient to improve the postural balance.
- Improper feedback from the ankle joint receptors could cause inappropriate motor reaction and poor balance control.

\section{Acknowledgements}

Rubens A. da Silva, was funded by the National Foundation for the Development of Private Higher Education (FUNADESP, Brazil). We gratefully acknowledge the assistance of Physiotherapist students for the recruitment of subjects.

\section{References}

1. Browne JE, O'Hare JN (2001) Review of different methods for assessing standing balance. Physiotherapy 87: 489-495.

2. Riemann B (2002) Is there a link between chronic ankle instability and posturalinstability. J Athl Train 37: 386-393.

3. Grigg P (1994) Peripheral neural mechanisms in proprioception. J Sport Rehabil 3: 2-17.

4. Nitz JC, Choy NL (2004) The relationship between ankle dorsiflexion range, falls and activity level in women aged 40 to 80 years. NZ Journal of Physiotherapy 32: $121-125$

5. Pope M, Chinn L, Mullineaux D, McKeon PO, Drewes L, et al. (2011) Spatia postural control alterations with chronic ankle instability. Gait Posture 34: 154158

6. McKeon PO, Hertel J (2008) Systematic Review of Postural Control and Latera Ankle Instability, Part I: Can Deficits Be Detected With Instrumented Testing? J Athl Train 43: 293-304.

7. Vaillant J, Rouland A, Martigné P, Braujou R, Nissen MJ, et al. (2009) Massage and mobilization of the feet and ankles in elderly adults: Effect on clinical balance performance. Man Ther 14: 661-664.

8. Vaillant J, Vuillerme N, Janvey A, Louis F, Braujou R, et al. (2008) Effect of manipulation of the feet and ankles on postural control in elderly adults. Research Bulletin 75: 18-22.

9. Winter DA (1995) Human balance and posture control during standing and walking. Gait Post 3: 193-214.

10. Nguyen DT, Kiel DP, Li W, Galica AM, Kang HG, et al. (2012) Correlations of clinical and laboratory measures of balance in older men and women. Arth Care Res 64: 1895-902.

11. López-Rodríguez S, Fernández de-Las-Peñas C, Alburquerque-Sendín F, Rodríguez-Blanco C, Palomeque-del-Cerro L (2007) Immediate Effects of manipulation of the talocrural joint on stabilometry and baropodometry in patients with anke sprain. J Manipulative Physiol Ther 30: 186-192.

12. Alburquerque-Sendín F, Fernández-de-las-Peñas $C$, Santos-del-Rey $M$ Martín-Vallejo FJ (2009) Immediate effects of bilateral manipulation of talocrura joints on standing stability in healthy subjects. Man Ther 14: 75-80.

13. Kapteyn TS, Bles W, Njiokiktjien CJ, Kodde L, Massen CH, et al. (1983) Standardization in platform stabilometry being a part of posturography. Agressologie 24: 321-332.

14. Santos BR, Delisle A, Larivière C, Plamondon A, Imbeau D (2008) Reliability of centre of pressure summary measures of postural steadiness in healthy young adults. Gait Posture 27: 408-415.

15. Lafond D, Corriveau H, Hébert R, Prince F (2004) Intrasession reliability of center of pressure measures of postural steadiness in healthy elderly people. Arch Phys Med Rehabil 85: 896-901.

16. Ruhe A, Fejer R, Walker B (2010) The test-retest reliability of centre of pressure measures in bipedal static task conditions - A systematic review of the literature. Gait Posture 32: 436-445.

17. da Silva RA, Bilodeau M, Parreira RB, Teixeira DC, Amorim CF (2013) Agerelated differences in time-limit performance and force platform-based balance measures during one-leg stance. J Electromyogr Kinesiol 23: v634-639.

18. Oliveira LF, Simpson DM and Nadal J (1996) Calculation of area of stabilometric signals using principal component analysis. Physiol Meas 17: 305-312.

19. Baratto L, Morasso PG, Re C, Spada G (2002) A new look at posturographic analysis in the clinical context: sway-density versus other parameterization techniques. Motor Control 6: 246-270.

20. Lee AJY, Lin WH, Huang $\mathrm{CH}$ (2006) Impaired proprioception and poor static 
Citation: Paes FJV, da Silva RA, Freira ALG, Politti F, Chiavegato L, et al. (2013) Immediate Effect of Bilateral Talocrural Joint Manipulation on Postural Balance in Healthy Subjects. J Ergonomics 3: 122. doi:10.4172/2165-7556.1000122

Page 5 of 5

postural control in subjects with functional instability of the ankle. J Exerc Sci Fit 4: 117-125

21. Burgess PR, Clark FJ (1969) Characteristics of knee joint receptors in the cat. J Physiol 203: 317-335.

22. Willems T, Witvrouw E, Verstuy J, Vaes P, Clercqet DD (2002) Proprioception and Muscle Strength in Subjects with a History of Ankle Sprains and Chronic Instability. J Athl Train 37: 487-493.

23. McKeon PO, Booi MJ, Branam B, Johnson DL, Mattacola CG (2010) Latera ankle ligament anesthesia significantly alters single limb. Gait Posture 32: 374 377.
Citation: Paes FJV, da Silva RA, Freira ALG, Politti F, Chiavegato L, et al. (2013) Immediate Effect of Bilateral Talocrural Joint Manipulation on Postural Balance in Healthy Subjects. J Ergonomics 3: 122. doi:10.4172/2165-7556.1000122
Submit your next manuscript and get advantages of OMICS Group submissions

Unique features:

User friendly/feasible website-translation of your paper to 50 world's leading languages Audio Version of published paper

Digital articles to share and explore

Special features:

300 Open Access Journals

25,000 editorial team

21 days rapid review proces

Quality and quick editorial, review and publication processing

Indexing at PubMed (partial), Scopus, EBSCO, Index Copernicus and Google Scholar etc

Sharing Option: Social Networking Enabled

Authors, Reviewers and Editors rewarded with online Scientific Credits

Better discount for your subsequent articles

Submit your manuscript at: www.omicsonline.org/submission 